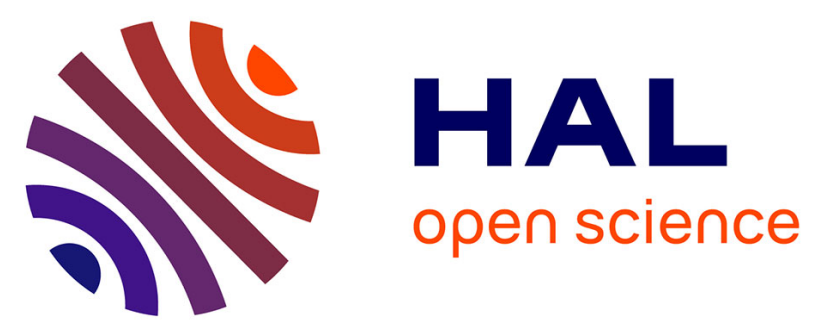

\title{
Derivation of a Macroscopic LWR Model from a Microscopic follow-the-leader Model by Homogenization
}

\author{
Nicolas Forcadel, Mamdouh Zaydan
}

\section{To cite this version:}

Nicolas Forcadel, Mamdouh Zaydan. Derivation of a Macroscopic LWR Model from a Microscopic follow-the-leader Model by Homogenization. 27th IFIP Conference on System Modeling and Optimization (CSMO), Jun 2015, Sophia Antipolis, France. pp.272-281, 10.1007/978-3-319-55795-3_25 . hal-01626910

\section{HAL Id: hal-01626910 https://hal.inria.fr/hal-01626910}

Submitted on 31 Oct 2017

HAL is a multi-disciplinary open access archive for the deposit and dissemination of scientific research documents, whether they are published or not. The documents may come from teaching and research institutions in France or abroad, or from public or private research centers.
L'archive ouverte pluridisciplinaire HAL, est destinée au dépôt et à la diffusion de documents scientifiques de niveau recherche, publiés ou non, émanant des établissements d'enseignement et de recherche français ou étrangers, des laboratoires publics ou privés.

\section{(c)(1)}

Distributed under a Creative Commons Attribution| 4.0 International License 


\title{
Derivation of a macroscopic LWR model from a microscopic follow-the-leader model by homogenization
}

\author{
Nicolas Forcadel and Mamdouh Zaydan \\ Normandie Université INSA Rouen, Laboratoire de Mathématiques de l'INSA de \\ Rouen, \\ Avenue de l'Université, 76800 Saint Etienne du Rouvray, France.
}

\begin{abstract}
The goal of this paper is to derive a traffic flow macroscopic model from a microscopic model with a transition function. At the microscopic scale, we consider a first order model of the form "follow the leader" i.e. the velocity of each vehicle depends on the distance to the vehicle in front of it. We consider two different velocities and a transition zone. The transition zone represents a local perturbation operated by a Lipschitz function. After rescaling, we prove that the "cumulative distribution function" of the vehicles converges towards the solution of a macroscopic homogenized Hamilton-Jacobi equation with a flux limiting condition at junction which can be seen as a LWR model.
\end{abstract}

\section{Introduction}

The goal of this paper is to present a rigorous derivation of a traffic flow macroscopic model by homogenization of a follow-the-leader model, see $[8,10]$. The idea is to rescale the microscopic model, which describes the dynamics of each vehicle individually, in order to get a macroscopic model which describes the dynamics of density of vehicles. Several studies have been done about the connection between microscopic and macroscopic traffic flow model. This type of connection is important since it allows us to deduce macroscopic models rigorously and without using strong assumptions. We refer for example to $[1,2,3]$ where the authors rescaled the empirical measure and obtained a scalar conservation law (LWR (Lighthill-Whitham-Richards) model). More recently, another kind of macroscopic models appears. These models rely on the Moskowitz function and make appear an Hamilton-Jacobi equation. This is the setting of our work which is a generalization of [6]. Indeed, authors in [6] considered a single road and one velocity throughout this road with a local perturbation at the origin while we consider two different velocities and a transition zone which can be seen as a local perturbation thats slows down the vehicles. At the macroscopic scale, we get an Hamilton-Jacobi equation with a junction condition at zero and an effective flux limiter. In order to have our homogenization result, we will construct the correctors. The main new technical difficulties comes from the construction of correctors and in particular the gradient estimates are more complicated from that in [6] because the gradient on the left and on the right may differ. 


\section{The microscopic model}

In this paper, we consider a "follow the leader" model of the following form

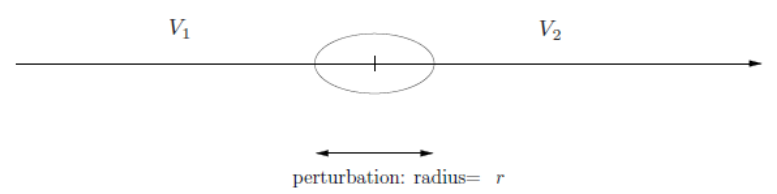

$$
\dot{U}_{j}(t)=V_{1}\left(U_{j+1}(t)-U_{j}(t)\right) \varphi\left(U_{j}(t)\right)+V_{2}\left(U_{j+1}(t)-U_{j}(t)\right)\left(1-\varphi\left(U_{j}(t)\right)\right),
$$

where $U_{j}$ denotes the position of the $j$-th vehicle and $\dot{U}_{j}$ its velocity. The function $\varphi$ simulates the presence of a local perturbation around the origin which allows us to pass from the optimal velocity function $V_{1}$ (on the left of the origin) to $V_{2}$ (on the right). We make the following assumptions on $V_{1}, V_{2}$ and $\varphi$.

\section{Assumption (A)}

- (A1) $V_{1}, V_{2}: \mathbb{R} \rightarrow \mathbb{R}^{+}$are Lipschitz continuous, non-negative and nondecreasing.

- (A2) For $i=1,2$, there exists a $h_{0}^{i} \in(0,+\infty)$ such that

$$
V_{i}(h)=0 \text { for all } h \leq h_{0}^{i} .
$$

- (A3) For $i=1,2$, there exists a $h_{\max }^{i} \in(0,+\infty)$ such that

$$
V_{i}(h)=V_{\text {imax }} \text { for all } h \geq h_{\max }^{i} .
$$

- (A4) For $i=1,2$, there exists a real $p_{0}^{i} \in\left[-1 / h_{0}^{i}, 0\right)$ such that the function $p \mapsto p V_{i}(-1 / p)$ is decreasing on $\left[-1 / h_{0}^{i}, p_{0}^{i}\right)$ and increasing on $\left[p_{0}^{i}, 0\right)$.

- (A5) The function $\varphi: \mathbb{R} \rightarrow[0,1]$ is Lipschitz continuous and

$$
\varphi(x)= \begin{cases}1 & \text { if } x \leq-r \\ 0 & \text { if } x>r\end{cases}
$$

\section{The homogenization result}

We introduce the "cumulative distribution function" of the vehicles:

$$
\rho(t, y)=-\left(\sum_{i \geq 0} H\left(y-U_{i}(t)\right)+\sum_{i<0}\left(-1+H\left(y-U_{i}(t)\right)\right)\right)
$$


and we make the following rescaling

$$
\rho^{\varepsilon}(t, y)=\varepsilon \rho(t / \varepsilon, y / \varepsilon) .
$$

$\rho^{\varepsilon}$ is a discontinuous solution of the following equation: for $(t, x) \in(0,+\infty) \times \mathbb{R}$,

$$
\left\{\begin{array}{l}
u_{t}^{\varepsilon}+\left(M_{1}^{\varepsilon}\left[\frac{u^{\varepsilon}(t, \cdot)}{\varepsilon}\right](x) \varphi\left(\frac{x}{\varepsilon}\right)+M_{2}^{\varepsilon}\left[\frac{u^{\varepsilon}(t, \cdot)}{\varepsilon}\right](x)\left(1-\varphi\left(\frac{x}{\varepsilon}\right)\right)\right) \cdot\left|u_{x}^{\varepsilon}\right|=0 \\
u^{\varepsilon}(0, x)=u_{0}(x)
\end{array}\right.
$$

where the non-local operators $M_{i}^{\varepsilon}$ and $M_{2}^{\varepsilon}$ are defined by

$$
M_{i}^{\varepsilon}[U](x)=\int_{-\infty}^{+\infty} J_{i}(z) E(U(x+\varepsilon z)-U(x)) d z-\frac{3}{2} V_{i \max }
$$

with

$$
E(z)=\left\{\begin{array}{l}
0 \text { if } z \geq 0 \\
1 / 2 \text { if }-1 \leq z<0, \quad J_{1}=V_{1}^{\prime} \text { and } J_{2}=V_{2}^{\prime} \text { on } \mathbb{R} . \\
3 / 2 \text { if } z<-1
\end{array}\right.
$$

We also assume that the initial condition satisfies the following assumption.

(A0) (Gradient bound). Let $k_{0}=\max \left(k_{0}^{1}, k_{0}^{2}\right)$ with $k_{0}^{i}=1 / h_{0}^{i}$. The function $u_{0}$ is Lipschitz continuous and satisfies

$$
-k_{0} \leq\left(u_{0}\right)_{x} \leq 0 .
$$

We have the following theorem (see [6]).

Theorem 1. Assume (AO) and (A). Then, there exists a unique viscosity solution $u^{\varepsilon}$ of (3.1). Moreover, the function $u^{\varepsilon}$ is continuous and there exists a constant $K$ such that

$$
u_{0}(x) \leq u^{\varepsilon}(t, x) \leq u_{0}(x)+K t .
$$

We will introduce now the macroscopic model which is a Hamilton-Jacobi equation on a junction. The Hamiltonians $\bar{H}_{1}$ and $\bar{H}_{2}$ are called effective Hamiltonians (see Proposition 2.9 in [6]) and are defined as follows: for $i=1,2$

$$
\bar{H}_{i}(p)= \begin{cases}-p-k_{0}^{i} & \text { for } p<-k_{0}^{i}, \\ -V_{i}\left(\frac{-1}{p}\right) \cdot|p| & \text { for }-k_{0}^{i} \leq p \leq 0, \\ p & \text { for } p>0\end{cases}
$$

with

$$
H_{0}^{i}=\min _{p \in \mathbb{R}} \bar{H}_{i}(p) \quad \text { and } H_{0}=\max \left(H_{0}^{1}, H_{0}^{2}\right) .
$$


Now we can define the limit problem. We refer to [9] for more details about existence and uniqueness of solution for this type of equation.

$$
\begin{cases}u_{t}^{0}+\bar{H}_{1}\left(u_{x}^{0}\right)=0 & \text { for } \quad(t, x) \in(0,+\infty) \times(-\infty, 0) \\ u_{t}^{0}+\bar{H}_{2}\left(u_{x}^{0}\right)=0 & \text { for } \quad(t, x) \in(0,+\infty) \times(0,+\infty) \\ u_{t}^{0}+F_{\bar{A}}\left(u_{x}^{0}\left(t, 0^{-}\right), u_{x}^{0}\left(t, 0^{+}\right)\right)=0 & \text { for } \quad(t, x) \in(0,+\infty) \times\{0\} \\ u^{0}(0, x)=u_{0}(x) & \text { for } \quad x \in \mathbb{R} .\end{cases}
$$

where $\bar{A}$ has to be determined and $F_{\bar{A}}$ is defined by

$$
F_{A}\left(p_{-}, p_{+}\right)=\max \left(\bar{A}, \bar{H}_{1}^{+}\left(p_{-}\right), \bar{H}_{2}^{-}\left(p_{+}\right)\right)
$$

$\bar{H}_{1}^{+}$and $\bar{H}_{2}^{-}$represent respectively the increasing and the decreasing part of $\bar{H}_{1}$ and $\bar{H}_{2}$. The following theorem is our main result in this paper.

Theorem 2. There exists $\bar{A} \in\left[H_{0}^{1}, 0\right]$ such that the function $u^{\varepsilon}$ defined by Theorem 1 converge locally uniformly towards the unique solution $u^{0}$ of (3.6).

Remark 1. Formally, if we derive (3.6), we will obtain a scalar conservation law with discontinuous flux whose literature is very rich, see for example [4]. However, the passage from microscopic to macroscopic models are more difficult in this setting and in particular on networks. On the contrary, the approach proposed in this paper can be extended to models posed on networks (see [5]).

\section{Correctors for the junction}

The key ingredient to prove the convergence result is to construct correctors for the junction. Given $\bar{A} \in \mathbb{R}$, we introduce two real numbers $\bar{p}_{1}, \bar{p}_{2} \in \mathbb{R}$, such that

$$
\bar{H}_{2}\left(\bar{p}_{2}\right)=\bar{H}_{2}^{+}\left(\bar{p}_{2}\right)=\bar{H}_{1}\left(\bar{p}_{1}\right)=\bar{H}_{1}^{-}\left(\bar{p}_{1}\right)=\bar{A} .
$$

If $\bar{A} \leq H_{0}$, we then define $\bar{p}_{1}, \bar{p}_{2} \in \mathbb{R}$ as the two real numbers satisfying

$$
\bar{H}_{2}\left(\bar{p}_{2}\right)=\bar{H}_{2}^{+}\left(\bar{p}_{2}\right)=\bar{H}_{1}\left(\bar{p}_{1}\right)=\bar{H}_{1}^{-}\left(\bar{p}_{1}\right)=H_{0} .
$$

Due to the form of $\bar{H}_{1}$ and $\bar{H}_{2}$ this two real numbers exist and are unique. We consider now the following problem: find $\lambda \in \mathbb{R}$ such that there exists a solution $w$ of the following global-in-time Hamilton-Jacobi equation

$$
\left(M_{1}[w](x) \cdot \varphi(x)+M_{2}[w](x) \cdot(1-\varphi(x))\right) \cdot\left|w_{x}\right|=\lambda \quad \text { for } x \in \mathbb{R}
$$

with

$$
M_{i}[U](x)=\int_{-\infty}^{+\infty} J_{i}(z) E(U(x+z)-U(x)) d z-\frac{3}{2} V_{i \max }
$$


Theorem 3 (Existence of a global corrector for the junction). Assume (A).

i) (General properties) There exists a constant $\bar{A} \in\left[H_{0}^{1}, 0\right]$ such that there exists a solution $w$ of (4.3) with $\lambda=\bar{A}$ and such that there exists a constant $C>0$ and a globally Lipschitz continuous function $m$ such that for all $x \in \mathbb{R}$,

$$
|w(x)-m(x)| \leq C .
$$

ii) (Bound from below at infinity) If $\bar{A}>H_{0}^{1}$, then there exists $\gamma_{0}$ such that for every $\gamma \in\left(0, \gamma_{0}\right)$, we have

$$
\left\{\begin{array}{l}
w(x-h)-w(x) \geq\left(-\bar{p}_{1}-\gamma\right) h-C \text { for } x \leq-r \text { and } h \geq 0, \\
w(x+h)-w(x) \geq\left(\bar{p}_{2}-\gamma\right) h-C \text { for } x \geq r \text { and } h \geq 0 .
\end{array}\right.
$$

iii) (Rescaling $w$ ) For $\varepsilon>0$, we set

$$
w^{\varepsilon}(x)=\varepsilon w\left(\frac{x}{\varepsilon}\right),
$$

then (along a subsequence $\varepsilon_{n} \rightarrow 0$ ) we have that $w^{\varepsilon}$ converges locally uniformly towards a function $W=W(x)$ which satisfies

$$
\begin{cases}|W(x)-W(y)| \leq C|x-y| & \text { for all } x, y \in \mathbb{R}, \\ \bar{H}_{1}\left(W_{x}\right)=\bar{A} & \text { for all } x<0, \\ \bar{H}_{2}\left(W_{x}\right)=\bar{A} & \text { for all } x>0 .\end{cases}
$$

In particular, we have (with $W(0)=0$ )

$$
W(x)=\bar{p}_{1} x 1_{\{x<0\}}+\bar{p}_{2} x 1_{\{x>0\}} .
$$

\section{$5 \quad$ Proof of Theorem 3}

This section contains the proof of Theorem 3. To do this, we will construct correctors on truncated domains and then pass to the limit as the size of the domain goes to infinity. For $l \in(r,+\infty), r<<l$ and $r \leq R<<l$, we want to find $\lambda_{l, R}$, such that there exists a solution $w^{l, R}$ of

$$
\begin{cases}Q_{R}\left(x,\left[w^{l, R}\right], w_{x}^{l, R}\right)=\lambda_{l, R} & \text { if } x \in(-l, l) \\ \bar{H}_{1}^{-}\left(w_{x}^{l, R}\right)=\lambda_{l, R} & \text { if } x \in\{-l\} \\ \bar{H}_{2}^{+}\left(w_{x}^{l, R}\right)=\lambda_{l, R} & \text { if } x \in\{l\},\end{cases}
$$

with

$$
\begin{aligned}
Q_{R}(x,[U], q) & =\psi_{R}(x) \cdot M_{2}[U](x) \cdot(1-\varphi(x)) \cdot|q|+\left(1-\psi_{R}(x)\right) \cdot \bar{H}_{2}(q) \\
& +\Phi_{R}(x) \cdot M_{1}[U](x) \cdot \varphi(x) \cdot|q|+\left(1-\Phi_{R}(x)\right) \cdot \bar{H}_{1}(q)
\end{aligned}
$$

and $\psi_{R}, \Phi_{R} \in C^{\infty}, \psi_{R}, \Phi_{R}: \mathbb{R} \rightarrow[0,1]$, with

$$
\psi_{R} \equiv\left\{\begin{array} { l l } 
{ 1 } & { x \leq R } \\
{ 0 } & { x > R + 1 }
\end{array} \quad \text { and } \quad \Phi _ { R } \equiv \left\{\begin{array}{ll}
1 & x \geq-R \\
0 & x<-R-1 .
\end{array}\right.\right.
$$


Proposition 1 (Existence of correctors on truncated domains). There exists a unique $\lambda_{l, R} \in \mathbb{R}$ such that there exists a solutions $w^{l, R}$ of (5.1). Moreover, there exists a constant $C$ (depending only on $k_{0}$ ), and a Lipschitz continuous function $m^{l, R}$, such that

$$
\left\{\begin{array}{l}
H_{0}^{1} \leq \lambda_{l, R} \leq 0, \\
\left|m^{l, R}(x)-m^{l, R}(y)\right| \leq C|x-y| \text { for } x, y \in[-l, l], \\
\left|w^{l, R}(x)-m^{l, R}(x)\right| \leq C \quad \text { for } x \in \mathbb{R} \times[-l, l] .
\end{array}\right.
$$

Proof. We only give the main steps of the proof. Classically, we will consider the approximated problem depending on the parameter $\delta$ and then take $\delta$ to 0 .

$$
\begin{cases}\delta v^{\delta}+Q_{R}\left(x,\left[v^{\delta}\right], v_{x}^{\delta}\right)=0 & \text { for } x \in(-l, l) \\ \delta v^{\delta}+\bar{H}_{1}^{-}\left(v_{x}^{\delta}\right)=0 & \text { for } x \in\{-l\} \\ \delta v^{\delta}+\bar{H}_{2}^{+}\left(v_{x}^{\delta}\right)=0 & \text { for } x \in\{l\}\end{cases}
$$

Step 1: construction of barriers .Using Perron's method and 0 and $\delta^{-1}\left|H_{0}^{1}\right|$ as barriers, we deduce that there exists a continuous viscosity solution $v^{\delta}$ of $(5.6)$ which satisfies

$$
0 \leq v^{\delta} \leq \frac{\left|H_{0}^{1}\right|}{\delta} .
$$

Step 2: control of the space oscillations of $v^{\delta}$. The function $v^{\delta}$ satisfies for all $x, y \in[-l, l], x \geq y$,

$$
-k_{0}(x-y)-1 \leq v^{\delta}(x)-v^{\delta}(y) \leq 0,
$$

with $k_{0}=\max \left(k_{0}^{1}, k_{0}^{2}\right)$ (see [6, Lemma 6.5]).

Step 3: construction of a Lipschitz estimate. As in [6, Lemma 6.6] we can construct a Lipschitz continuous function $m^{\delta}$, such that there exists a constant C, (independent of $l, R$ and $\delta$ ) such that

$$
\begin{cases}\left|m^{\delta}(x)-m^{\delta}(y)\right| \leq C|x-y| & \text { for all } x, y \in[-l, l], \\ \left|v^{\delta}(x)-m^{\delta}(x)\right| \leq C & \text { for all } x \in[-l, l] .\end{cases}
$$

Step 4: passing to the limit as $\delta$ goes to 0 . Classicly, taking $\delta$ to zero, we get $\lambda_{l . R}, w^{l, R}$ and $m^{l, R}$ satisfiying (5.5). The uniqueness of $\lambda_{l, R}$ is classical so we skip it. This ends the proof of Proposition 1.

Proposition 2. The following limits exist (up to a subsequence)

$$
\bar{A}_{R}=\lim _{l \rightarrow+\infty} \lambda_{l, R}, \quad \text { and } \quad \bar{A}=\lim _{R \rightarrow+\infty} \bar{A}_{R} .
$$

Moreover, we have

$$
H_{0}^{1} \leq \bar{A}_{R}, \bar{A} \leq 0
$$


Proposition 3 (Control of the slopes on a truncated domain). Assume that $l$ and $R$ are big enough. Let $w^{l, R}$ be the solution of (5.1) given by Proposition 1. We also assume that up to a sub-sequence $\bar{A}=\lim _{R \rightarrow+\infty} \lim _{l \rightarrow+\infty} \lambda_{l, R}>H_{0}^{1}$. Then there exits a $\gamma_{0}>0$ such that for all $\gamma \in\left(0, \gamma_{0}\right)$, there exists a constant $C$ (independent of $l$ and $R$ ) such that for all $x \leq-r$ and $h \geq 0$

$$
w^{l, R}(x-h)-w^{l, R}(x) \geq\left(-\bar{p}_{1}-\gamma\right) h-C .
$$

Similarly, for all $x \geq r$ and $h \geq 0$,

$$
w^{l, R}(x+h)-w^{l, R}(x) \geq\left(\bar{p}_{2}-\gamma\right) h-C .
$$

Proof. We only prove (5.9) since the proof for (5.10) is similar. For $\sigma>0$ small enough, we denote by $p_{-}^{\sigma}$ the real number such that

$$
\bar{H}_{1}\left(p_{-}^{\sigma}\right)=\bar{H}_{1}^{-}\left(p_{-}^{\sigma}\right)=\lambda_{l, R}-\sigma .
$$

Let us now consider the function $w^{-}=p_{-}^{\sigma} x$ that satisfies

$$
\bar{H}_{1}\left(w_{x}^{-}\right)=\lambda_{l, R}-\sigma \quad \text { for } x \in \mathbb{R} .
$$

We also have

$$
M_{1}\left[w^{-}\right](x)=-V_{1}\left(\frac{-1}{p_{-}^{\sigma}}\right) .
$$

For all $x \in(-l,-r)$, using that $\varphi(x)=1$ and $\psi_{R}(x)=1$, we deduce that $w^{-}$ satisfies

$$
\begin{cases}Q_{R}\left(x,\left[w^{-}\right], w_{x}^{-}\right)=\lambda_{l, R}-\mu & \text { for } x \in(-l,-r) \\ \bar{H}_{1}^{-}\left(w_{x}^{+}\right)=\lambda_{l, R}-\mu & \text { for } x \in\{-l\} .\end{cases}
$$

Using the comparaison principle, we deduce that for all $h \geq 0$, for all $x \in$ $(-l,-r)$, we have that

$$
w^{l, R}(x-h)-w^{l, R}(x) \geq-p_{-}^{\sigma} h-2 C .
$$

Finally, for $\gamma_{0}$ and $\sigma$ small enough, we can set $p_{-}^{\sigma}=\bar{p}_{1}+\gamma$.

Proof of Theorem 3. The proof is performed in two steps.

Step 1: proof of $i$ ) and ii). The goal is to pass to the limit as $l \rightarrow+\infty$ and then as $R \rightarrow+\infty$. There exists $l_{n} \rightarrow+\infty$, such that

$$
m^{l_{n}, R}-m^{l_{n}, R}(0) \rightarrow m^{R} \text { as } n \rightarrow+\infty,
$$

the convergence being locally uniform. We also define

$$
\begin{aligned}
& \bar{w}^{R}(x)=\limsup _{l_{n} \rightarrow+\infty}\left(w^{l_{n}, R}-w^{l_{n}, R}(0)\right), \\
& \underline{w}^{R}(x)=\liminf _{l_{n} \rightarrow+\infty_{*}}\left(w^{l_{n}, R}-w^{l_{n}, R}(0)\right) .
\end{aligned}
$$


Thanks to (5.5), we know that $\bar{w}^{R}$ and $\underline{w}^{R}$ are finite and satisfy

$$
m^{R}-C \leq \underline{w}^{R} \leq \bar{w}^{R} \leq m^{R}+C .
$$

By stability of viscosity solutions, $\bar{w}^{R}-2 C$ and $\underline{w}^{R}$ are respectively a sub and a super-solution of

$$
Q_{R}\left(x,\left[w^{R}\right], w_{x}^{R}\right)=\bar{A}_{R} \quad \text { for } x \in \mathbb{R}
$$

Therefore, using Perron's method, we can construct a solution $w^{R}$ of (5.11) with $m^{R}, \bar{A}^{R}$ and $w^{R}$ satisfying

$$
\begin{cases}\left|m^{R}(x)-m^{R}(y)\right| \leq C|x-y| & \text { for all } x, y \in \mathbb{R} \\ \left|w^{R}(x)-m^{R}(x)\right| \leq C & \text { for } x \in \mathbb{R} \times \mathbb{R} \\ H_{0}^{1} \leq \bar{A}_{R} \leq 0 & \end{cases}
$$

Using Proposition 3 , if $\bar{A}>H_{0}$, we know that there exists $\gamma_{0}$ and $C>0$, such that for all $\gamma \in\left(0, \gamma_{0}\right)$,

$$
\left\{\begin{array}{l}
w^{R}(x-h)-w^{R}(x) \geq\left(-\bar{p}_{1}-\gamma\right) h-C \text { for all } x \leq-r, h \geq 0, \\
w^{R}(x+h)-w^{R}(x) \geq\left(\bar{p}_{2}-\gamma\right) h-C \text { for all } x \geq r, h \geq 0
\end{array}\right.
$$

Passing to the limit as $R \rightarrow+\infty$ and proceeding as above, the proof is complete.

Step 2: proof of iii). Using (4.6), we have that

$$
w^{\varepsilon}(x)=\varepsilon m\left(\frac{x}{\varepsilon}\right)+O(\varepsilon) .
$$

Therefore, we can find a sequence $\varepsilon_{n} \rightarrow 0$, such that

$$
w^{\varepsilon_{n}} \rightarrow W \text { locally uniformly as } n \rightarrow+\infty,
$$

with $W(0)=0$. Like in $[7]($ Appendix A.1), we have that

$$
\bar{H}_{1}\left(W_{x}\right)=\bar{A} \quad \text { for } x<0 \text { and } \quad \bar{H}_{2}\left(W_{x}\right)=\bar{A} \quad \text { for } x>0 .
$$

For all $\gamma \in\left(0, \gamma_{0}\right)$, we have that if $\bar{A}>H_{0}^{1}$ and $x>0$,

$$
W_{x} \geq \bar{p}_{2}-\gamma,
$$

where we have used (4.6). Therefore we get

$$
W_{x}=\bar{p}_{2} \text { for } x>0,
$$

Similarly, we get $W_{x}=\bar{p}_{1}$ for $x<0$. This ends the proof of Theorem 3 . 


\section{Proof of convergence}

In this section, we will prove our homogenization result. Classicly, the proof relies on the existence of correctors. We will just prove the convergence result at the junction point since at any other point, the proof is classical using that $v=0$ is a corrector, see [6].

Proof of Theorem 2. We introduce

$$
\bar{u}(t, x)=\limsup _{\varepsilon \rightarrow 0} u^{\varepsilon} \quad \text { and } \quad \underline{u}(t, x)=\liminf _{\varepsilon \rightarrow 0} u^{\varepsilon} .
$$

Let us prove that $\bar{u}$ is a sub-solution of (3.6) at the point 0 , (the proof for $\underline{u}$ is similar and we skip it). The definition of viscosity solution for Hamilton-Jacobi equation is presented in Section 2 in [9]. We argue by contradiction and assume that there exist a test function $\Psi \in \mathcal{C}^{1}\left(J_{\infty}\right)$ such that

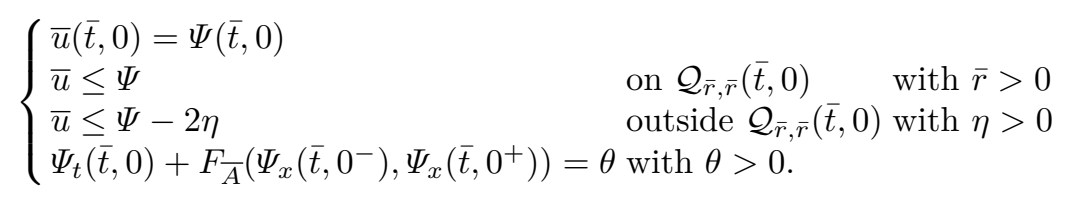

According to [9], we may assume that the test function has the following form

$$
\Psi(t, x)=g(t)+\bar{p}_{1} x 1_{\{x<0\}}+\bar{p}_{2} x 1_{\{x>0\}} \quad \text { on } \mathcal{Q}_{\bar{r}, 2 \bar{r}}(\bar{t}, 0),
$$

The last line in condition (6.2) becomes

$$
g^{\prime}(\bar{t})+F_{\bar{A}}\left(\bar{p}_{1}, \bar{p}_{2}\right)=g^{\prime}(\bar{t})+\bar{A}=\theta .
$$

Let us consider $w$ the solution of (4.3) provided by Theorem 3, and let us denote

$$
\Psi^{\varepsilon}(t, x)= \begin{cases}g(t)+w^{\varepsilon}(x) & \text { on } \mathcal{Q}_{\bar{r}, 2 \bar{r}}(\bar{t}, 0) \\ \Psi(t, x) & \text { outside } \mathcal{Q}_{\bar{r}, 2 \bar{r}}(\bar{t}, 0) .\end{cases}
$$

We claim that $\Psi^{\varepsilon}$ is a viscosity solution on $Q_{\bar{r}, \bar{r}}(\bar{t}, 0)$ of the following problem,

$$
\Psi_{t}^{\varepsilon}+\left(\tilde{M}_{1}^{\varepsilon}\left[\frac{\Psi^{\varepsilon}}{\varepsilon}(t, \cdot)\right](x) \varphi\left(\frac{x}{\varepsilon}\right)+\tilde{M}_{2}^{\varepsilon}\left[\frac{\Psi^{\varepsilon}}{\varepsilon}(t, \cdot)\right](x)\left(1-\varphi\left(\frac{x}{\varepsilon}\right)\right)\right) \cdot\left|\Psi_{x}^{\varepsilon}\right| \geq \frac{\theta}{2} .
$$

Indeed, let $h$ be a test function touching $\varphi^{\varepsilon}$ from below at $\left(t_{1}, x_{1}\right) \in \mathcal{Q}_{\bar{r}, \bar{r}}(\bar{t}, 0)$, so we have that the function $\chi(y)=\frac{1}{\varepsilon}\left(h\left(t_{1}, \varepsilon y\right)-g\left(t_{1}\right)\right)$ touches $w$ from below at $\frac{x_{1}}{\varepsilon}$ which implies that

$$
\left(\tilde{M}_{1}[w]\left(\frac{x_{1}}{\varepsilon}\right) \varphi\left(\frac{x_{1}}{\varepsilon}\right)+\tilde{M}_{2}[w]\left(\frac{x_{1}}{\varepsilon}\right)\left(1-\varphi\left(\frac{x_{1}}{\varepsilon}\right)\right)\right) \cdot\left|h_{x}\left(t_{1}, x_{1}\right)\right| \geq \bar{A} .(6.6
$$

Using (6.4) and the fact that $h_{t}\left(t_{1}, x_{1}\right)=g^{\prime}\left(t_{1}\right)$ and computing (6.6), we get the desired result. 
Getting the contradiction. We have that for $\varepsilon$ small enough

$$
u^{\varepsilon}+\eta \leq \Psi=g(t)+\bar{p}_{1} x 1_{\{x<0\}}+\bar{p}_{2} x 1_{\{x>0\}} \quad \text { on } \mathcal{Q}_{\bar{r}, 2 \bar{r}}(\bar{t}, 0) \backslash \mathcal{Q}_{\bar{r}, \bar{r}}(\bar{t}, 0) .
$$

Using the fact that $w^{\varepsilon} \rightarrow W$, and using (4.8), we have for $\varepsilon$ small enough

$$
u^{\varepsilon}+\frac{\eta}{2} \leq \Psi^{\varepsilon} \quad \text { on } \mathcal{Q}_{\bar{r}, 2 \bar{r}}(\bar{t}, 0) \backslash \mathcal{Q}_{\bar{r}, \bar{r}}(\bar{t}, 0) .
$$

Combining this with (6.5), we get that

$$
u^{\varepsilon}+\frac{\eta}{2} \leq \Psi^{\varepsilon} \text { outside } \mathcal{Q}_{\bar{r}, \bar{r}}(\bar{t}, 0) .
$$

By the comparison principle on bounded subsets the previous inequality holds in $\mathcal{Q}_{\bar{r}, \bar{r}}(\bar{t}, 0)$. Passing to the limit as $\varepsilon \rightarrow 0$ and evaluating the inequality at $(\bar{t}, 0)$, we obtain the following contradiction

$$
\bar{u}(\bar{t}, 0)+\frac{\eta}{2} \leq \Psi(\bar{t}, 0)=\bar{u}(\bar{t}, 0) .
$$

\section{ACKNOWLEDGMENTS}

This work is co-financed by the European Union with the European regional development fund (ERDF, HN0002137) and by the Normandie Regional Council via the M2NUM project.

\section{References}

1. A. Aw, A. Klar, M. Rascle, and T. Materne, Derivation of continuum traffic flow models from microscopic follow-the-leader models, SIAM Journal on Applied Mathematics, 63 (2002), pp. 259-278.

2. R. M. Colombo And E. Rossi, Rigorous estimates on balance laws in bounded domains, Acta Mathematica Scientia, 35 (2015), pp. 906-944.

3. M. Di Francesco AND M. D. Rosini, Rigorous derivation of the lighthillwhitham-richards model from the follow-the-leader model as many particle limit, arXiv preprint arXiv:1404.7062, (2014).

4. S. DIEHL, On scalar conservation laws with point source and discontinuous flux function, SIAM journal on mathematical analysis, 26 (1995), pp. 1425-1451.

5. N. Forcadel AND W. Salaza, Homogenization of a discrete model for a bifurcation and application to traffic flow.

6. N. ForCADEL AND W. SAlazAR, A junction condition by specified homogenization of a discrete model with a local perturbation and application to traffic flow, $<$ hal01097085>, (2015).

7. G. Galise, C. Imbert, and R. Monneau, A junction condition by specified homogenization, arXiv preprint arXiv:1406.5283, (2014).

8. D. C. Gazis, R. Herman, and R. Rothery, Nonlinear follow-the-leader models of traffic flow, Operations Research, 9 (1961), pp. 545-567.

9. C. Imbert AND R. MonNeAu, Flux-limited solutions for quasi-convex hamiltonjacobi equations on networks, <hal-00832545>, (2014).

10. I. Prigogine And R. Herman, Kinetic theory of vehicular traffic, (1971), p. 100. 